\title{
OUTAGE ANALYSIS OF SIMO SYSTEM OVER NAKAGAMI-N FADING CHANNEL
}

\author{
Preeti Sonker ${ }^{1}$, Himanshu Katiyar ${ }^{2}$, Ashawani Kumar ${ }^{3}$ \\ ${ }^{1}$ M.Tech, Department of Electronics and Communication Engineering, School of Engineering, BBDU, Lucknow, India \\ ${ }^{2}$ M.Tech, Department of Electronics and Communication Engineering, School of Engineering, BBDU, Lucknow, India \\ ${ }^{3}$ M.Tech, Department of Electronics and Communication Engineering, School of Engineering, BBDU, Lucknow, India
}

\begin{abstract}
In this paper, we present the performance analysis of multi-antenna system over Nakagami-n fading channel in wireless communication. Here, firstly we have analyzed the system performance such as probability density function (PDF), and outage probability single antenna system and after then SIMO system over Nakagami-n fading channel.

Nakagami-n fading channel is a very popular channel which has its basis on its experimental or mathematical observation. To improve the system performance of the channel and also increase the received SNR (signal to noise ratio), We are using multiple numbers of antennas (using diversity techniques such as selection combining (SC)) at the receiver. In this paper, we have derived the closed form expression for PDF of SNR, and outage probability over Nakagami-n fading channel.
\end{abstract}

Keywords: Nakagami-n Fading channel, PDF, Outage probability (Pout), Selection combining (SC). $* * *$

\section{INTRODUCTION}

Motivated by the importance of Nakagami-n statistical models to describe the channel fading in land, terrestrial, and satellite telecommunication. In recent year, the idea of wireless communication channel has received a lot of attention by researchers. In wireless communication [1], multiple propagation path exist from transmitter to receiver due to reflection, diffraction and scattering of wave by different objects. Signal copies received through different path can undergo rapid attenuation, distortion, delays and phase shift over a short period of time due to constructive or destructive interference occurring at receiver. In destructive interference signal power decreases significantly and this phenomenon is called as 'fading' [2]. The multiple propagation path are time-varying. The time varying nature of channel is the major constraint for reliable wireless transmission. Multipath fading results in severe performance degradation in terms of outage probability and bit error rate in wireless communication system. The nature of radio propagation environment describes the statistical behavior of radio signals.

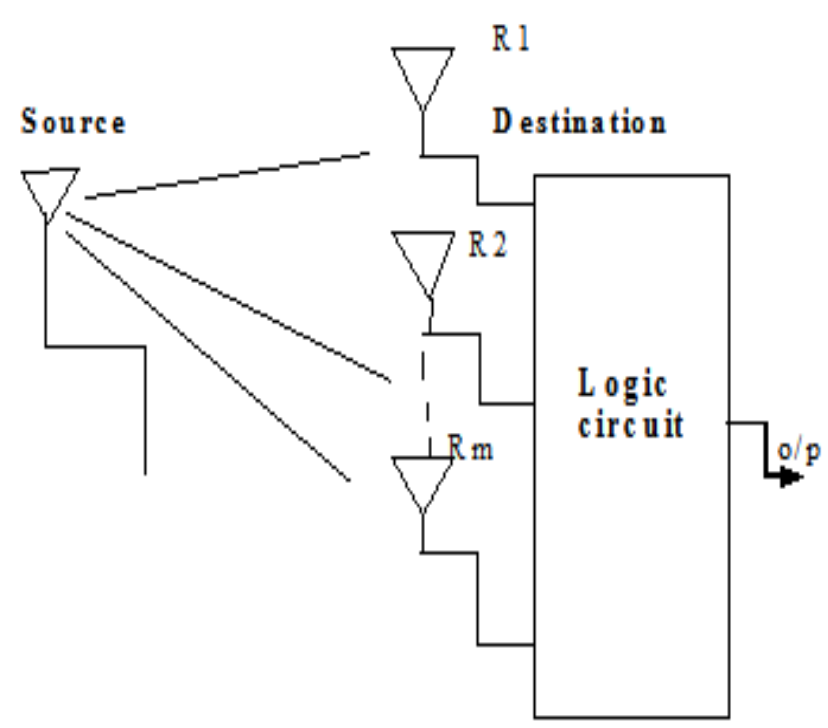

Fig.1 SIMO system using SC technique

There are various distributions, which describe the statistics of the fading channel. Long-term signal variation is characterized by log normal distribution. The short term distribution is described by several distributions such as Rayleigh, Rician, and Nakagami-n distribution [3]. The single most effective technique to accomplish reliable communication over a wireless channel is 'diversity' techniques which provide the receiver with independent faded copies of the transmitted signal with the hope that at least one of the copy received correctly. In this paper, we are using selection diversity (SC) technique to derive the closed form expression of outage probability. Outage probability [4] is defined as the probability that defines the received SNR 
remains below a threshold value of SNR. Firstly we have analyzed the outage probability (OP) for single transmitter and single receiver system but sometimes this system could not decrease the OP. The probability density function has been derived in [5], over Nakagami-n fading channel.

As shown in fig. (2), we can see that if signal to noise ratio remains below the threshold level, then the region is called faded region and if the value of SNR goes across the threshold level, then the region is called the non-faded region [6]. In non-faded region, the error probability becomes very low or negligible.

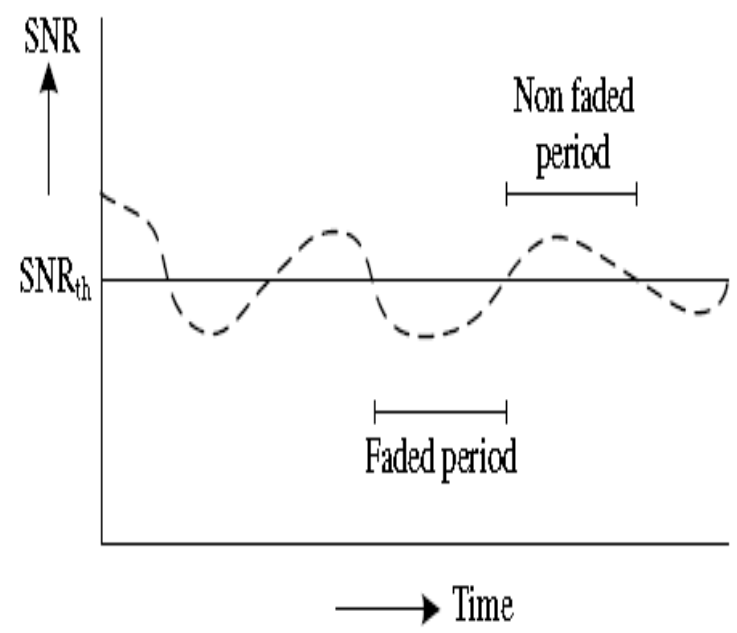

Fig.2 SNR vs Time

As shown in fig. (2), we can see that if signal to noise ratio remains below the threshold level, then the region is called faded region and if the value of SNR goes across the threshold level, then the region is called the non-faded region [6]. In non-faded region, the error probability becomes very low or negligible.

To the best of our knowledge, no one has derived exact closed form expression for the OP and BER over Nakagami$\mathrm{n}$ fading channel. The Nakagami-n fading channel is a very popular channel model. It is also known as the Rician channel. It is used to model propagation paths consisting of one strong direct line of sight (LOS) component and many other weaker components. Nakagami-n fading channel is typically observed in the first resolvable LOS paths of microcellular urban and suburban land-mobile, picocellular indoor and factory environments. It also is used to model the dominant LOS path of satellite, microwave and ship to ship radio links [10]. Hence, for reducing the fading and error probability we are using multiple numbers of antennas in receiving end of the receiver, as shown in fig.(1).

\section{SYSTEM MODEL}

Firstly we consider a single transmitter at the source (S), and single receiver at the destination (D). If a signal is transmitted from the transmitter to the receiver over a wireless communication link, then the received signal power may become very low and signal may be faded due to noise and multiple obstructions in open environment. Hence, for increasing the received signal strength, we will have to increase the SNR. But sometimes it does not possible through single antenna system, so that we are using multiple numbers of antennas as shown in fig. (1). Let us denote the transmitter by $\mathrm{T}$ and receiver by $\mathrm{R} 1, \mathrm{R} 2 \ldots \mathrm{Rm}$. Here we have to derive the simplest expression for both OP and PDF. The SNR is denoted by the running variable gamma $(\gamma)$. A multi-antenna system receives signal from source through multiple links and performs selection combining. After SC, if received SNR is above the threshold of the decoder, the message is decoded and retransmitted to the destination in the next time slot. The multi-antenna system of selection diversity technique is used to improve the system performance over Nakagami-n fading channel. In selection diversity, a logic circuit is used to select the best one value of SNR.

\section{PERFORMANCE ANALYSIS}

\subsection{Mathematical Modeling of Outage Probability}

The SNR per symbol of the Nakagami-n channel, $\gamma$, is distributed according to a Non-central chi square as [10]...

$$
P_{\gamma}(\gamma)=\frac{\left(1+n^{2}\right) e^{-n^{2}}}{\bar{\gamma}} e^{\frac{-\left(1+n^{2}\right) \gamma}{\bar{\gamma}}} I_{o}\left(2 n \sqrt{\left.\frac{\left(1+n^{2}\right) \gamma}{\bar{\gamma}}\right)}\right.
$$

$\gamma \geq 0,(1)$

where $P_{\gamma}(\gamma)$ is the probability density function (PDF) of received SNR and is the Nakagami-n fading parameter, which takes values from 0 to $\infty$ (from Rayleigh fading channel to constant channel) and $K=n^{2}$ is Nakagami-n Kfactor which is the ratio between power of the LOS component to the average power of scattered components and bar $\gamma$, is average SNR per symbol. $I_{o}(z)$ is the zeroth order modified Bessel function of first kind.

To simplify the probability distribution function (PDF) of this channel, we use the infinite series representation of Bessel function which is defined as below [9]

$$
I_{o}(z)=\sum_{k=0}^{\infty} \frac{\left(\frac{z}{2}\right)^{2}}{(k !)^{2}}
$$

Using equation (1) and (2), we can be written as

$$
P_{\gamma}(\gamma)=\frac{\left(1+n^{2}\right) e^{-n^{2}}}{\bar{\gamma}} \sum_{k=0}^{\infty} \frac{\left(n^{2 k}\right)\left(\frac{1+n^{2}}{\bar{\gamma}}\right)^{k}}{(k !)^{2}}\left[e^{\frac{-\left(1+n^{2}\right) \gamma}{\bar{\gamma}}} \gamma^{k}\right]
$$

Equation (3), is the simplifying PDF of received SNR over Nakagami-n fading channel. Here $P_{\gamma}(\gamma)$ is defined as the PDF. 
Now, the outage probability is denoted by the $P_{\text {out }}$, can be expressed as below

$$
\text { Pout }=\int_{0}^{\gamma / h} p_{\gamma}(\gamma) d \gamma
$$

To evaluate the cumulative distribution function, (CDF), we have to integrate the equation (3) from 0 to threshold value of received SNR.

\section{NUMERICAL RESULT}

This section presents the PDF of SNR and Outage probability of system in which source is communicating with destination, supported by multi-antenna $\mathrm{Rm}$. To verify the numerical result, we use the MatLab tool and plot the graphical representation of PDF, Bessel function and Outage probability. In this system, we consider the value of SNR in between 0 to 50 for PDF and the fading parameter-n have taken from 1 to 3 . Here $\mathrm{K}=\mathrm{n}^{2}$ is the Rician $\mathrm{K}$-factor and the value of $\mathrm{K}$ have taken from 0 to 100 . In the case of PDF, we converse the both PDF from equation (1) and (2) and we see that both PDF converse properly. Now, we sure about that we can use the simplify PDF from equation (3) for further implementation. After then, we plot the graph on Outage probability for MISO system over Nakagami-n fading channel and have taken the value of SNR from 0 to 10 and value of $\mathrm{K}$ from 0 to 10 .

Fig. (3) Shows the both PDF graph representation i.e. converse properly. In fig (4), we can see that the graph on outage probability w.r.t SNR and we got the result that as we increase the value of SNR, hence the value of Pout becomes less.

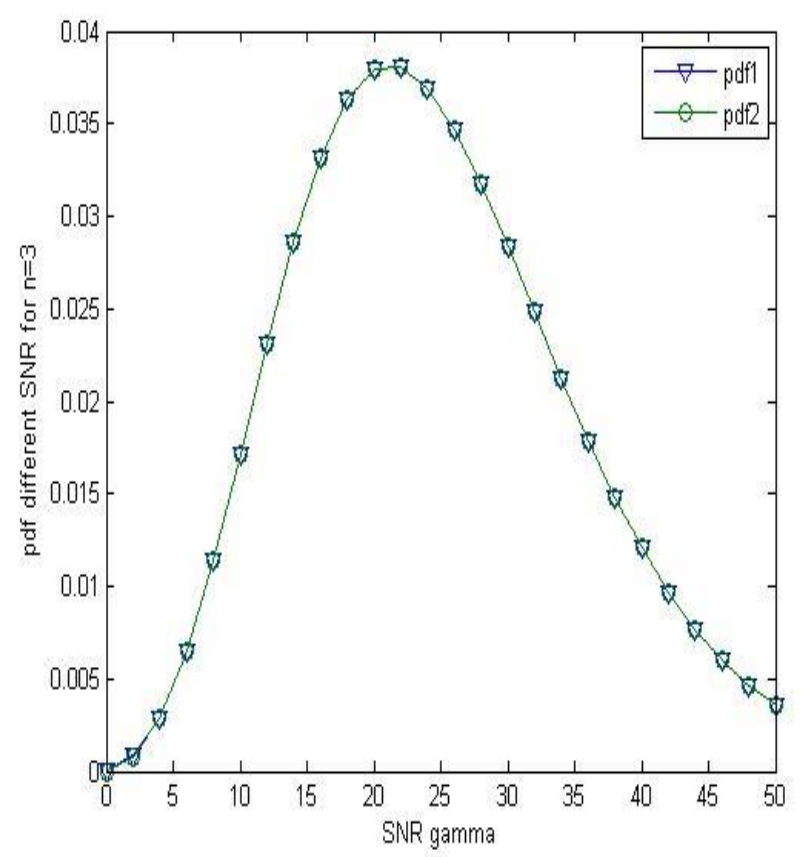

Fig 3 Probability density function vs SNR over Nakagami-n fading channel.



Fig. 4 outage probability vs SNR over Nakagami-n fading channel

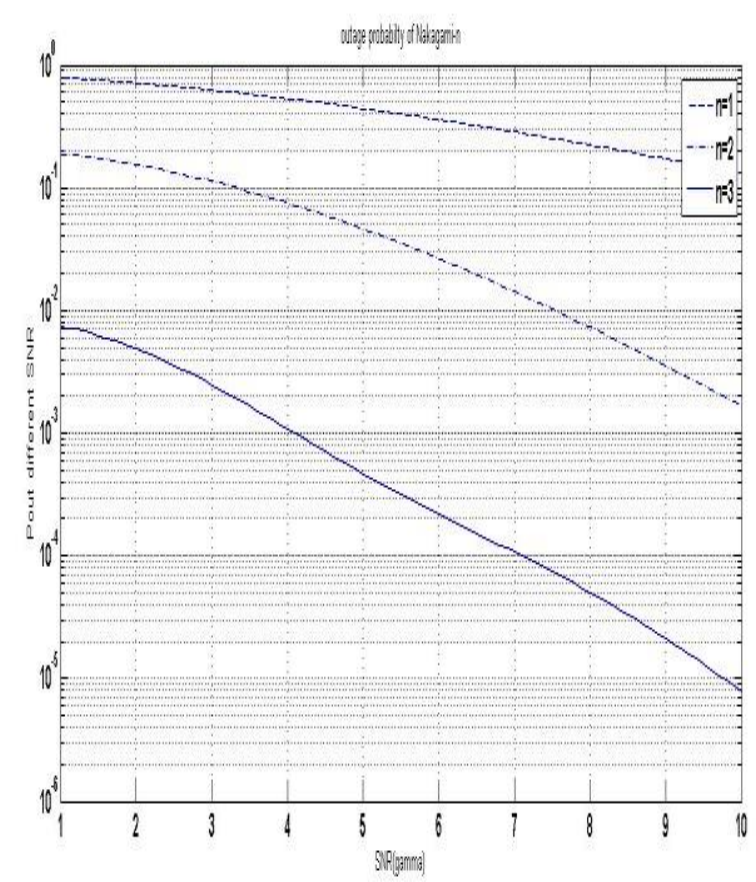

Fig. 5 outagr probabilty vs SNR w.r.t different fading parameter n over Nakagami-n fading channel.

\section{CONCLUSIONS}

In this paper, we have analyzed the outage performance analysis of MISO system over Nakagami-n fading channel in wireless communication system. We deirved the numerical and graphical result of probability density function PDF and Outage probability Pout w.r.t to running variable gamma (SNR). We can see that however as we increase the value of SNR then the Pout level becomes less. Hence we can reduce the faded region in the wireless communication system. 


\section{REFERENCES}

[1]. H. Katiyar and R. Bhattacharjee, "Performance of TwoHop Regenerative Relay Network under Correlated Nakagami-m Fading at Multi-antenna Relay," IEEE Commun.. Lett., vol. 13, no. 10, Oct. 2009 (scheduledto be published).

[2]. Mohammad Rakibul Islam 1, Jinsang Kim 2 Dept. of Electronics and Radio Engineering Kyung Hee University1 Seocheon, Kihung, Yongin, Gyeonggi, 449-701,

[3]. Dimitris A. Zogas, Member, IEEE, George K. Karagiannidis, Senior Member, IEEE, and A.

[4]. Wiley.IEEE.PRESS. (Digital Communication Over Fading Channel-2 ${ }^{\text {nd }}$ Edition)

[5]. Table of Integrals, Series, and Product, Seventh Edition.

[6]. Rappaport, T.S., Wireless Communication: Principles and Practice, Prentice Hall 1996.

[7]. M. Dohler and Y. Li, Cooperative communication: Hardware, channel and PHY. John Wiley \& Sons, 2010.

[8]. A. Bletsas, A. Khisti, D. Reed, and A. Lippman, "A simple cooperative diversity method based on network path selection," IEEE J. Select. Areas Commun., vol. 24, no. 3, pp. 659-672, March 2006.

[9]. N. C. Beaulieu and J. Hu, "A closed-form expression for the outage Probability of decode-and-forward relaying in dissimilar rayleigh fading Channels," IEEE Commun. Lett., vol. 10, no. 12, pp. 813 -815, December 2006.

[10]. J. Hu and N. Beaulieui, "Performance analysis of decode-and-forward relaying with selection combining," IEEE Commun. Lett., vol.11, no. 6, pp. 489 -491, june 2007.

[11]. N. Sagias, F. Lazarakis, G. Tombras, and C. Datsikas, "Outage analysis of decode-and-forward relaying over nakagami- $m$ fading channels," IEEE Signal Processing Lett., vol. 15, pp. 41-44, 2008.

[12]. T. Duong, V. N. Q. Bao, and H. j. Zepernick, "On the performance of selection decode-and-forward relay networks over nakagami-m fading channels," IEEE Commun. Lett., vol. 13, no. 3, pp. 172 -174, Mar. 2009.

[13]. S. S. Ikki and M. H. Ahmed, "Performance analysis of adaptive decode- and-forward cooperative diversity networks with best-relay selection," IEEE Trans. Commun., vol. 58, no. 1, pp. 68-72, Jan. 2010.

[14]. Y. Lee, M. hung Tsai, and S. lan Sou, "Performance of decode-and- forward cooperative communications with multiple dual-hop relays over nakagami-m fading channels," IEEE Trans. Wireless Commun., vol. 8, no. 6, pp. 2853-2859, Jun 2009.

[15]. M. Di Renzo, F. Graziosi, and F. Santucci, "On the performance of csi-assisted cooperative communications over generalized fading channels," in Communications, 2008. ICC '08. IEEE International Conference on, May 2008, pp. 1001 $-1007$.

[16]. M. Simon and M. Alouini, Digital Communications Over Fading Channels. Wiley \& Sons, INC., Publication, 2005.

[17]. S. L. M. R. Speigel and J. Liu, Mathematical Handbook of formulas and tables, schaums outline series, 3rd edition. M. G. Hill, 2009.

[18]. M. Elkashlan, P. L. Yeoh, C. Sung, and I. Collings, "Distributed multi-antenna relaying in nonregenerative cooperative networks," in Personal Indoor and Mobile Radio
Communications (PIMRC), 2010 IEEE 21 $1^{\text {st }}$ International Symposium on, Sep 2010, pp. $24-29$.

[19]. Y. A. B. A. P. Prudnikov and O. I. Marichev, Integrals and Series.

Volume 4: Direct Laplace Transforms. CRC publisher, 1992. [20]. M. K. Simon and M. S. Alouini, "Digital Communication over Fading Channels," 2 ed. New York: Wiley, 2005.

[21]. Y. Zhao, R. Adve,"Outage probability at arbitrary SNR with cooperativediversity," IEEE Commun. Lett., vol. 9, pp. 700-702, AUG. 2005.

[22]. G. Taricco and E. Biglieri,"Exact pairwise error probability of spacetime codes," IEEE Trans. Inform. Theory, vol. 48, pp. 510513, Feb. 2002.

[23]. W. H. Tranter, K. S. Shanmugan, T. S. Rappaport, K. L. Kosbar, "Principles of communication systems simulation with wireless applications." Prentice Hall, 2004, pp. 266 269.

[24]. M. S. Alouini, A. J. Goldsmith, “A unified approach for calculating error rates of linearlymodulated signals over generalized fading channels," IEEE Trans. Commun., vol. 47, pp. 1324-1334, Sep. 1999.

[25]. Y.C. Ko, M. S. Alouini, M. K. Simon,’Outage Probability of Diversity Systems over Generalized Fading Channels," IEEE Trans. Commun., vol. 48, pp. 1783-1787, NOV. 2000. 